\title{
Sarnak Awarded 2014 Wolf Prize
}

The 2014 Wolf Prize in Mathematics has been awarded to Peter SARnak of Princeton University and the Institute for Advanced Study "for his deep contributions to analysis, number theory, geometry, and combinatorics."

\section{Description of the Prizewinner's Work}

Following is the citation from the Wolf Foundation.

Peter Sarnak is a mathematician covering an extremely broad spectrum with a far-reaching vision. He has influenced the development of several mathematical fields, often by uncovering deep and unsuspected connections. In analysis, he investigated eigenfunctions of quantum mechanical Hamiltonians which correspond to chaotic classical dynamical systems in a series of fundamental papers. He formulated and supported the quantum unique ergodicity conjecture, asserting that all eigenfunctions of the Laplacian on negatively curved manifolds are uniformly distributed in phase space. Sarnak's introduction of tools from number theory into this domain allowed him to obtain results which had seemed out of reach and paved the way for much further progress, in particular the recent works of E. Lindenstrauss and N. Anantharaman. In his work on $L$-functions (jointly with Z. Rudnick) the relationship of contemporary research on automorphic forms to random matrix theory and the Riemann hypothesis is brought to a new level by the computation of higher correlation functions of the Riemann zeros. This is a major step forward in the exploration of the link between random matrix theory and the statistical properties of zeros of the Riemann zeta function going back to H. Montgomery and A. Odlyzko. In 1999 it culminated in the fundamental work, jointly with N. Katz, on the statistical properties of low-lying zeros of families of L-functions. Sarnak's work (with A. Lubotzky and R. Philips) on Ramanujan graphs had a huge impact on combinatorics and computer science. Here again he used deep results in number theory to make surprising and important advances in another discipline.

By his insights and his readiness to share ideas he has inspired the work of students and fellow researchers in many areas of mathematics.

\section{Biographical Sketch}

Peter Sarnak was born in Johannesburg, South Africa, in 1953. He received his Ph.D. from Stanford University in 1980 under the direction of Paul Cohen.
He has held positions at the Courant Institute of Mathematical Sciences, New York University (1980-1983 and 2001-2005); Stanford University (1984-1991); Princeton University (1991-1999 and 2002-present); and the Institute for Advanced Study (member, 1999-2002, 20052007; professor, 2007-present). He is currently the Eugene Higgins Professor of Mathematics at Princeton University, as well as a professor at the Institute for Advanced Study in Princeton.

Sarnak held an Alfred P. Sloan Foundation Fellowship from 1983 to 1985. He was chosen as a Presidential Young Investigator (1985-1990). He was awarded the Polya Prize of the Society of Industrial and Applied Mathematics (SIAM) in 1998 and the

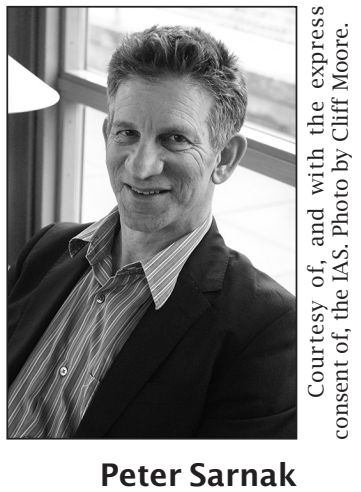
Ostrowski Prize in 2001. In 2003, Sarnak and his coauthor Nicholas Katz received the AMS Conant Prize for their article "Zeroes of zeta functions and symmetry", which appeared in the Bulletin of the AMS 36 (1999), 1-26. Sarnak also received the AMS Cole Prize in Number Theory in 2005 for his fundamental contributions to number theory and, in particular, his book Random Matrices, Frobenius Eigenvalues and Monodromy, written jointly with Katz. He was awarded the Lester Ford Prize of the Mathematical Association of America (MAA) in 2012. He has been elected to the American Academy of Arts and Sciences (1991), the National Academy of Sciences (2002), and the American Philosophical Society (2008). He was elected a fellow of the Royal Society of London in 2002.

\section{About the Prize}

The Wolf Prize carries a cash award of US $\$ 100,000$. The science prizes are given annually in the areas of agriculture, chemistry, mathematics, medicine, and physics. Laureates receive their awards from the president of the State of Israel in a special ceremony at the Knesset building (Israel's parliament) in Jerusalem. The list of previous recipients of the Wolf Prize in Mathematics is available on the website of the Wolf Foundation, http://www.wolffund.org.i1.

DOI: http://dx.doi.org/10.1090/noti1122 